\title{
Increasing the Bitlength of a Crypto-Coprocessor
}

\author{
Wieland Fischer and Jean-Pierre Seifert \\ Infineon Technologies \\ Security $\&$ ChipCard ICs \\ CC TI Concepts 86 Innovations \\ D-81609 Munich \\ Germany \\ \{wieland.fischer, jean-pierre.seifert\}@infineon.com
}

\begin{abstract}
We present a novel technique which allows a virtual increase of the bitlength of a crypto-coprocessor in an efficient and elegant way. The proposed algorithms assume that the coprocessor is equipped with a special modular multiplication instruction. This instruction, called MultModDiv $(A, B, N)$ computes $A * B \bmod N$ and $\lfloor(A * B) / N\rfloor$. In addition to the doubling algorithm, we also present two conceivable economic implementations of the MultModDiv instruction: one hardware and one software realization. The hardware realization of the MultModDiv instruction has the same performance as the modular multiplication presented in the paper. The software realization requires two calls of the modular multiplication instruction. Our most efficient algorithm needs only six calls to an $n$-bit MultModDiv instruction to compute a modular $2 n$-bit multiplication. Obviously, special variants of our algorithm, e.g., squaring, require fewer calls.
\end{abstract}

Keywords: Arithmetical coprocessor, Hardware architecture, Modular multiplication, Hardware/Software codesign.

\section{Introduction}

Fast modular multiplication algorithms have been extensively studied $\mathrm{Ba} \mid \mathrm{DQ}$, HP1 HP2 Knu Mo OmPai Q Sed WQWa. This is due to the fact that large integer arithmetic is essential for public-key cryptography. Recently, we have seen some progress of integer factorization $\left[\mathrm{C}^{+}\right.$which demands for higher RSA bit lengths. On the other hand, for low cost and low power devices (e.g., in Smartcards, PDAs, Cellular Phones, etc.) one has to use hardware which does not provide sufficient bitlengths.

Unfortunately, these two requirements lead to a burden of the system issuer, e.g., the card industry. The source of this burden is the fact that, say 2048-bit RSA, cannot be handled efficiently on a 1024 bit device. Only with some workaround this problem becomes a manageable task. Namely, as it is now commonly known, one can use the Chinese Remainder Theorem for the RSA signature, see 
$[\mathrm{CQ}]$. To keep the RSA verification also relatively simple, most often the fourth Fermat number is used as public exponent. Only recently it was shown how to efficiently reduce such modular 2048-bit multiplications to 1024-bit modular multiplications, see [HP1|HP2 Pai]. Pailler Pai] initiated this doubling research topic and formulated the following research problem:

Problem. Find an $n k$-bit modular multiplication algorithm using a minimal number of $n$-bit modular operations.

His algorithm needs nine modular multiplications for the case $k=2$, see Pai]. In this paper we will provide an answer to his question by presenting a novel doubling algorithm. Our most general and most efficient algorithm needs only six $n$-bit modular operations to compute a $2 n$-bit modular multiplication. The idea for our family of algorithms is based on the fact that the coprocessor is equipped with a special modular multiplication instruction. This instruction is called MultModDiv and defined within the next section. An optimal realization is clearly achieved using an enhanced hardware modular multiplication instruction. Nevertheless, an efficient software realization of this instruction is possible. The software realization requires two calls to the modular multiplication instruction. Both realizations of this MultModDiv instruction will be presented.

The present paper is organized as follows: The next section gives the necessary definitions of MultModDiv. Section 3 explains our basic doubling algorithm, an enhanced version and also our special purpose variants. In section 4 we introduce the simple software emulation of the MultModDiv instruction. Finally, in section 5 we show how to realize the MultModDiv instruction in hardware.

\section{Preliminaries}

\subsection{The Instructions MultMod and MultModInit $\boldsymbol{n}_{\boldsymbol{n}}$}

The following definition is the usual modular multiplication.

Definition 1. For numbers $A, B$ and $N, N>0$, the MultMod instruction is defined as

$$
R=\operatorname{MultMod}(A, B, N)
$$

with

$$
R:=(A * B) \bmod N .
$$

The following extension of the modular multiplication is already a feature of today's existing crypto coprocessors.

Definition 2. For a fixed integer $n$ and numbers $A, B, C$ and $N, N>0$, the MultModInit $_{n}$ instruction is defined as

$$
R=\operatorname{MultModInit}_{n}(A, B, C, N)
$$

with

$$
R:=\left(A * B+C * 2^{n}\right) \bmod N .
$$




\subsection{The Instructions MultModDiv and MultModDivInit $\boldsymbol{n}_{\boldsymbol{n}}$}

The following definition is a natural extension of the usual modular multiplication.

Definition 3. For a fixed integer $n$ and numbers $A, B$ and $N, N>0$, the MultModDiv is defined as

$$
(Q, R)=\operatorname{MultModDiv}(A, B, N)
$$

with

$$
Q:=\left\lfloor\frac{A * B}{N}\right\rfloor \quad \text { and } \quad R:=(A * B)-Q * N
$$

Definition 4. For a fixed integer $n$ and numbers $A, B, C$ and $N, N>0$, the

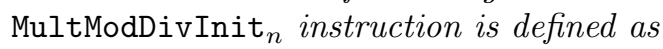

$$
(Q, R)=\text { MultModDivInit }_{n}(A, B, C, N)
$$

with

$$
Q:=\left\lfloor\frac{A * B+C * 2^{n}}{N}\right\rfloor \quad \text { and } \quad R:=\left(A * B+C * 2^{n}\right)-Q * N .
$$

\section{The Doubling Algorithm}

\subsection{Modular Multiplication without Initialization}

We will start with the easiest of our algorithms, which needs 7 MultModDiv instructions on an $n$-bit processor.

Theorem 1. There exists an algorithm to compute $A * B \bmod N$ using seven MultModDiv instructions of length $n$, provided that $2^{2 n-1} \leq N<2^{2 n}$ and $0 \leq$ $A, B<N$.

Proof. We will first present the algorithm. 


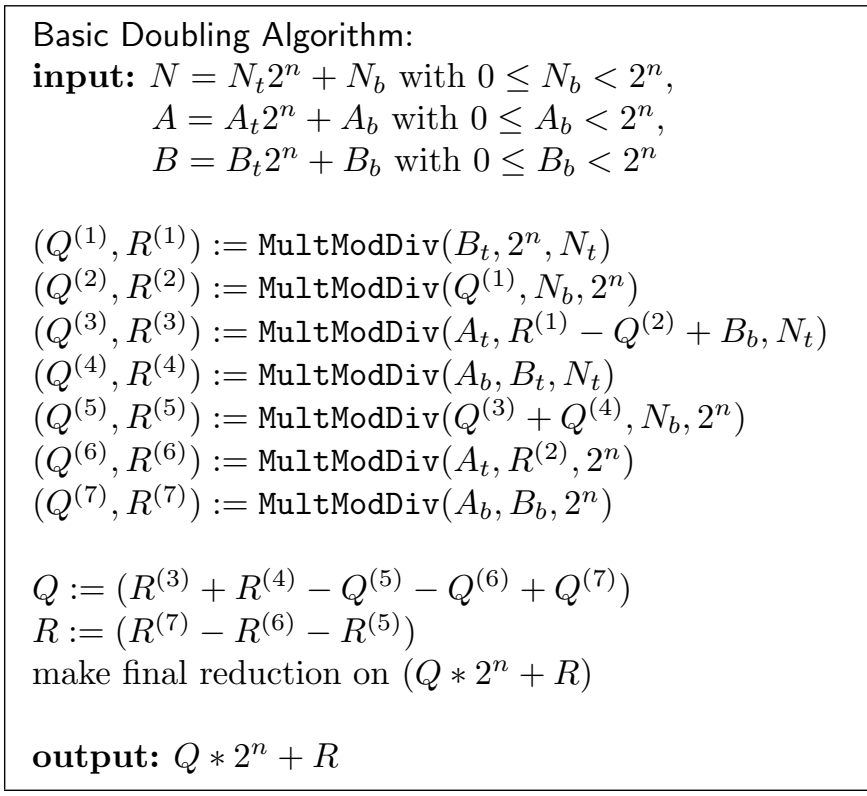

We will prove that $\left(R^{(3)}+R^{(4)}-Q^{(5)}-Q^{(6)}+Q^{(7)}\right) * 2^{n}+\left(R^{(7)}-R^{(6)}-R^{(5)}\right)$ is indeed congruent to $A * B$ modulo $N$. This can easily be seen from the following, where we use $Z=2^{n}$ as abbreviation.

$$
\begin{aligned}
& \left(A_{t} Z+A_{b}\right) *\left(B_{t} Z+B_{b}\right) \\
= & A_{t} B_{t} Z Z+A_{t} B_{b} Z+A_{b} B_{t} Z+A_{b} B_{b} \\
= & A_{t}\left(Q^{(1)} N_{t}+R^{(1)}\right) Z+A_{t} B_{b} Z+A_{b} B_{t} Z+A_{b} B_{b} \\
\equiv & A_{t} R^{(1)} Z-A_{t} Q^{(1)} N_{b}+A_{t} B_{b} Z+A_{b} B_{t} Z+A_{b} B_{b} \\
= & A_{t} R^{(1)} Z-A_{t}\left(Q^{(2)} Z+R^{(2)}\right)+A_{t} B_{b} Z+A_{b} B_{t} Z+A_{b} B_{b} \\
= & A_{t}\left(R^{(1)}-Q^{(2)}+B_{b}\right) Z-A_{t} R^{(2)}+A_{b} B_{t} Z+A_{b} B_{b} \\
= & \left(Q^{(3)} N_{t}+R^{(3)}\right) Z-A_{t} R^{(2)}+A_{b} B_{t} Z+A_{b} B_{b} \\
= & \left(Q^{(3)} N_{t}+R^{(3)}\right) Z-A_{t} R^{(2)}+\left(Q^{(4)} N_{t}+R^{(4)}\right) Z+A_{b} B_{b} \\
\equiv & \left(R^{(3)}+R^{(4)}\right) Z-\left(Q^{(3)}+Q^{(4)}\right) N_{b}-A_{t} R^{(2)}+A_{b} B_{b} \\
= & \left(R^{(3)}+R^{(4)}\right) Z-\left(Q^{(5)} Z+R^{(5)}\right)-A_{t} R^{(2)}+A_{b} B_{b} \\
= & \left(R^{(3)}+R^{(4)}\right) Z-\left(Q^{(5)} Z+R^{(5)}\right)-\left(Q^{(6)} Z+R^{(6)}\right)+A_{b} B_{b} \\
= & \left(R^{(3)}+R^{(4)}\right) Z-\left(Q^{(5)} Z+R^{(5)}\right)-\left(Q^{(6)} Z+R^{(6)}\right)+\left(Q^{(7)} Z+R^{(7)}\right) \\
= & \left(R^{(3)}+R^{(4)}-Q^{(5)}-Q^{(6)}+Q^{(7)}\right) Z+\left(R^{(7)}-R^{(6)}-R^{(5)}\right) \bmod N
\end{aligned}
$$

The two congruences above are based on the fact that $N_{t} Z \equiv-N_{b} \bmod N$. Apart from the fact that this result still has to be reduced modulo $N$, this completes the proof. 


\section{Practical Implementation Issues}

1. Observe that in steps three and five negative numbers may occur. This can be resolved by the fact that for positive numbers $A, B$ and $N$ the equation $(Q, R)=\operatorname{MultModDiv}(A, B, N)$ implies $(-Q-1, N-R)=$ $\operatorname{MultModDiv}(A,-B, N)$, if $R \neq 0$.

2. It is possible that the intermediary output $(Q, R)$ is not reduced, i.e., $0 \leq$ $R<2^{n}$ and $0 \leq Q<N_{t}$ is not fulfilled. In this case one has to do a final reduction: first, do $(Q, R) \leftarrow\left(Q \pm N_{t}, R \pm N_{b}\right)$ until $Q$ is reduced modulo $N_{t}$. Then, do $(Q, R) \leftarrow\left(Q \pm 1, R \mp 2^{n}\right)$ until $R$ is reduced modulo $2^{n}$.

3 . Using two parallel $n$-bit processors one only needs the time of four MultModDiv instructions.

4. If the given module $N$ has an odd bitlength, then one has to compute with $2 * N$.

\subsection{Modular Multiplication with Initialization}

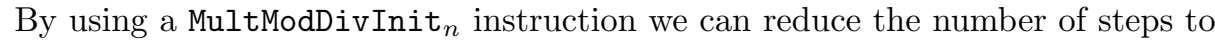
six.

Theorem 2. There exists an algorithm to compute $A * B \bmod N$ using five MultModDiv and one MultModDivInit ${ }_{n}$ instruction of length $n$, provided that $2^{2 n-1} \leq N<2^{2 n}$ and $0 \leq A, B<N$.

Proof. We first present the algorithm.

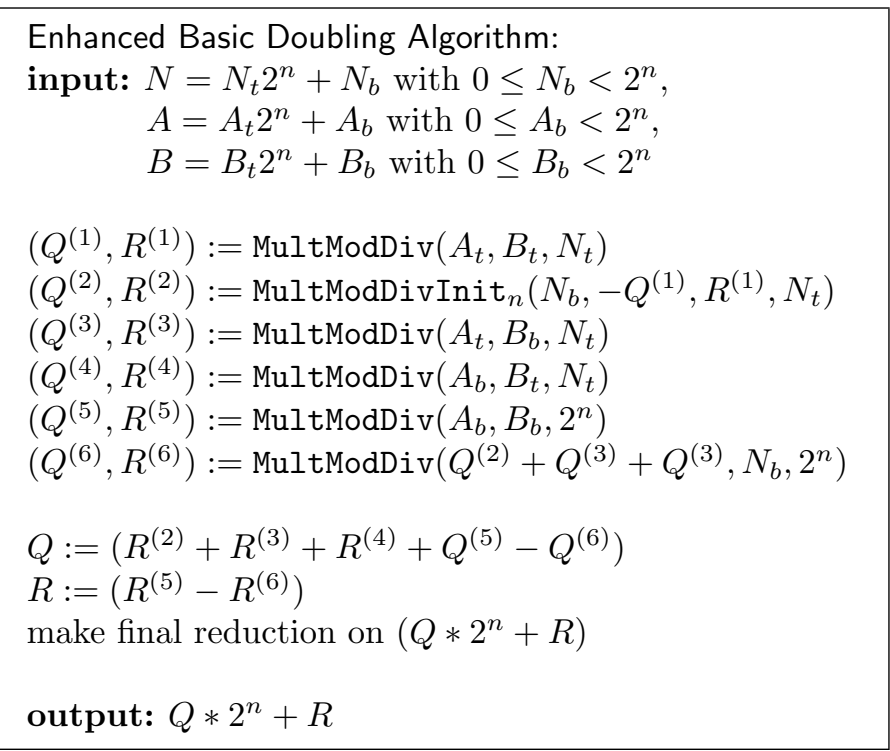

We will prove that $\left(R^{(2)}+R^{(3)}+R^{(4)}+Q^{(5)}-Q^{(6)}\right) * 2^{n}+\left(R^{(5)}-R^{(6)}\right)$ is indeed congruent to $A * B$ modulo $N$. This can be seen from the following, where we use $Z=2^{n}$ as abbreviation. 


$$
\begin{aligned}
& \left(A_{t} Z+A_{b}\right) *\left(B_{t} Z+B_{b}\right) \\
= & A_{t} B_{t} Z Z+A_{t} B_{b} Z+A_{b} B_{t} Z+A_{b} B_{b} \\
= & \left(Q^{(1)} N_{t}+R^{(1)}\right) Z Z+A_{t} B_{b} Z+A_{b} B_{t} Z+A_{b} B_{b} \\
\equiv & \left(R^{(1)} Z-Q^{(1)} N_{b}\right) Z+A_{t} B_{b} Z+A_{b} B_{t} Z+A_{b} B_{b} \\
= & \left(Q^{(2)} N_{t}+R^{(2)}\right) Z+A_{t} B_{b} Z+A_{b} B_{t} Z+A_{b} B_{b} \\
\equiv & \left(R^{(2)} Z-Q^{(2)} N_{b}\right)+A_{t} B_{b} Z+A_{b} B_{t} Z+A_{b} B_{b} \\
= & \left(R^{(2)} Z-Q^{(2)} N_{b}\right)+\left(Q^{(3)} N_{t}+R^{(3)}\right) Z+A_{b} B_{t} Z+A_{b} B_{b} \\
= & \left(R^{(2)} Z-Q^{(2)} N_{b}\right)+\left(Q^{(3)} N_{t}+R^{(3)}\right) Z+\left(Q^{(4)} N_{t}+R^{(4)}\right) Z+A_{b} B_{b} \\
= & \left(R^{(2)} Z-Q^{(2)} N_{b}\right)+\left(Q^{(3)} N_{t}+R^{(3)}\right) Z+\left(Q^{(4)} N_{t}+R^{(4)}\right) Z+\left(Q^{(5)} Z+R^{(5)}\right) \\
\equiv & \left(R^{(2)}+R^{(3)}+R^{(4)}+Q^{(5)}\right) Z-\left(Q^{(2)}+Q^{(3)}+Q^{(4)}\right) N_{b}+R^{(5)} \\
= & \left(R^{(2)}+R^{(3)}+R^{(4)}+Q^{(5)}\right) Z-\left(Q^{(6)} Z+R^{(6)}\right)+R^{(5)} \\
= & \left(R^{(2)}+R^{(3)}+R^{(4)}+Q^{(5)}-Q^{(6)}\right) Z+\left(R^{(5)}-R^{(6)}\right) \bmod N
\end{aligned}
$$

The three congruences above are based on the fact that $N_{t} Z \equiv-N_{b} \bmod N$. Apart from the fact that this result still has to be reduced modulo $N$, this completes the proof.

\section{Practical Implementation Issues}

1. Observe that in steps two and six negative numbers may occur. This can be resolved as shown above.

2. It is possible that the intermediary output $(Q, R)$ is not reduced. This can be resolved as shown above.

3. Using two parallel $n$-bit processors one only needs the time of three MultModDiv instructions.

4. Again, if the given module $N$ has an odd bitlength, then one has to compute with $2 * N$.

\subsection{Optimized Special Purpose Variants}

Now the basic strategy of our algorithms should be clear. Therefore, we will present the results for special purpose variants.

\section{Squaring}

Theorem 3. There exists an algorithm to compute $A^{2} \bmod N$ using six MultModDiv instructions of length $n$, provided that $2^{2 n-1} \leq N<2^{2 n}$ and $0 \leq A<N$.

If we consider the algorithm of section 3.2 for the case $A=B$, we see that steps three and four are identical. Therefore, we get the following result:

Theorem 4. There exists an algorithm to compute $A^{2} \bmod N$ using four MultModDiv and one MultModDivInit ${ }_{n}$ instruction of length $n$, provided that $2^{2 n-1} \leq N<2^{2 n}$ and $0 \leq A<N$. 


\section{Precomputation}

If the factor $B$ is known in advance (e.g., square and multiply for exponentiation), then the first and second computation of the algorithm of section 3.1 can be carried out in advance. Therefore, the multiplication can be done in five steps.

Theorem 5. There exists an algorithm to compute $A * B \bmod N$ using five MultModDiv instructions of length $n$, provided that $2^{2 n-1} \leq N<2^{2 n}$ and $0 \leq$ $A, B<N$, where $B$ is known in advance.

Using a completely different idea, one needs only six MultModDiv steps. This time, one uses a special representation of $A$ and $B$. Namely, $A=A_{t} * N_{t}+A_{b}$ and $B=B_{t} * N_{t}+B_{b}$, where $N_{t}:=\lfloor\sqrt{N}\rfloor$.

Theorem 6. There exists an algorithm to compute $A * B \bmod N$ using six MultModDiv instructions of length $n$, provided that $2^{2 n-1} \leq N<2^{2 n}$ and $0 \leq A, B<N$, where $N$ is known in advance..

\section{Software Realization of the MultModDiv and MultModDivInit ${ }_{n}$ Instructions}

This section presents a software emulation of the MultModDiv and

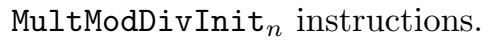

Theorem 7. There exists an algorithm to compute $\operatorname{MultModDiv}(A, B, N)$ using two MultMod instructions, provided that $0 \leq A, B<N$.

Proof. We present the simple algorithm.

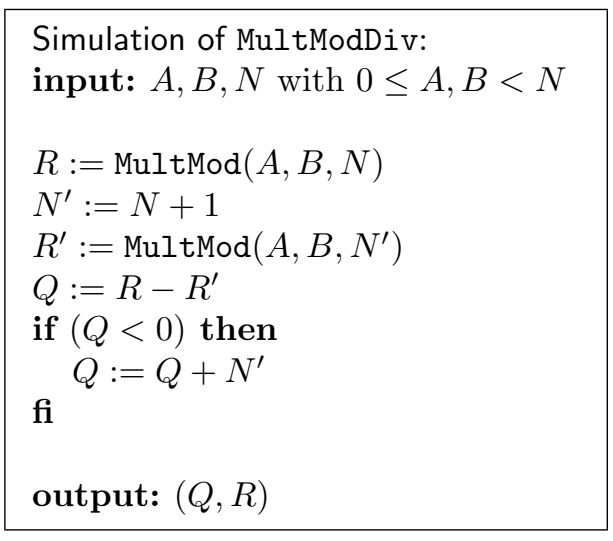

We will prove that the former algorithm correctly computes the MultModDiv instruction. For given inputs $A, B$ and $N$ there exists some $Q$ and $R$ with

$$
A * B=Q * N+R \text { and } R=(A * B) \bmod N,
$$

where $0 \leq R<N$ and $0 \leq Q<N-1$. Equivalently, we also have

$$
A * B=Q *(N+1)+(R-Q) .
$$


For $(R-Q) \geq 0$ this means $(R-Q)=(A * B) \bmod (N+1)$ and for $(R-Q)<0$ this means $(R-Q)+(N+1)=(A * B) \bmod (N+1)$. Thus, for $Q$ we have

$$
Q=((A * B) \bmod N)-((A * B) \bmod (N+1))
$$

or, if this value is less than zero

$$
Q=((A * B) \bmod N)-((A * B) \bmod (N+1))+(N+1)
$$

This completes the proof.

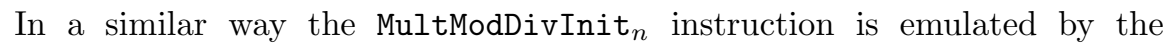

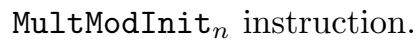

Theorem 8. There exists an algorithm to compute MultModDivInit ${ }_{n}$

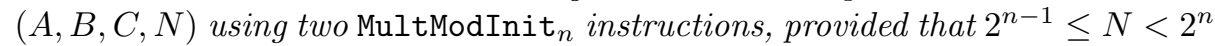
and $0 \leq A, B, C<N$.

Proof. We present the algorithm.

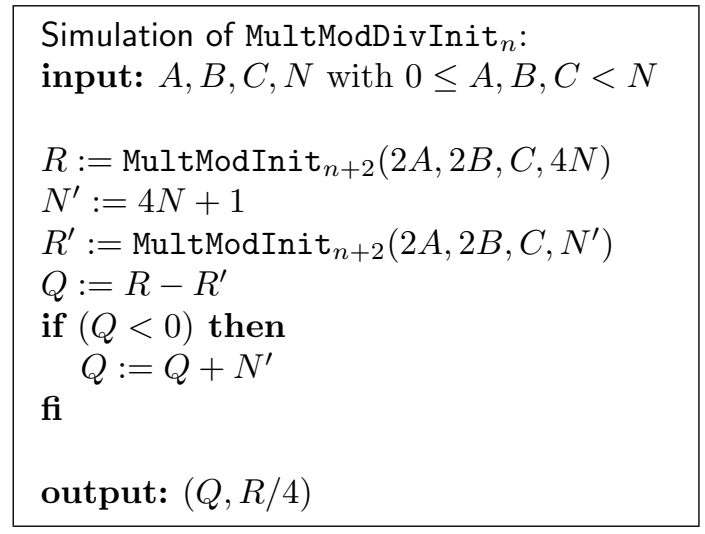

The proof is a derivation of the former one, leaving the modifications to the reader. However, we note that bounding the size of the quotient $Q$ is the crucial point.

Both algorithms can be extended to algorithms also working for non-reduced $A, B$ and $C$. This is necessary for our doubling algorithms.

\section{Hardware Realization of the MultModDiv and MultModDivInit ${ }_{n}$ Instructions}

We will now sketch how an algorithm for the MultMod instruction can be extended into an algorithm for the MultModDiv instruction. We first consider the textbook MultMod implementation. 


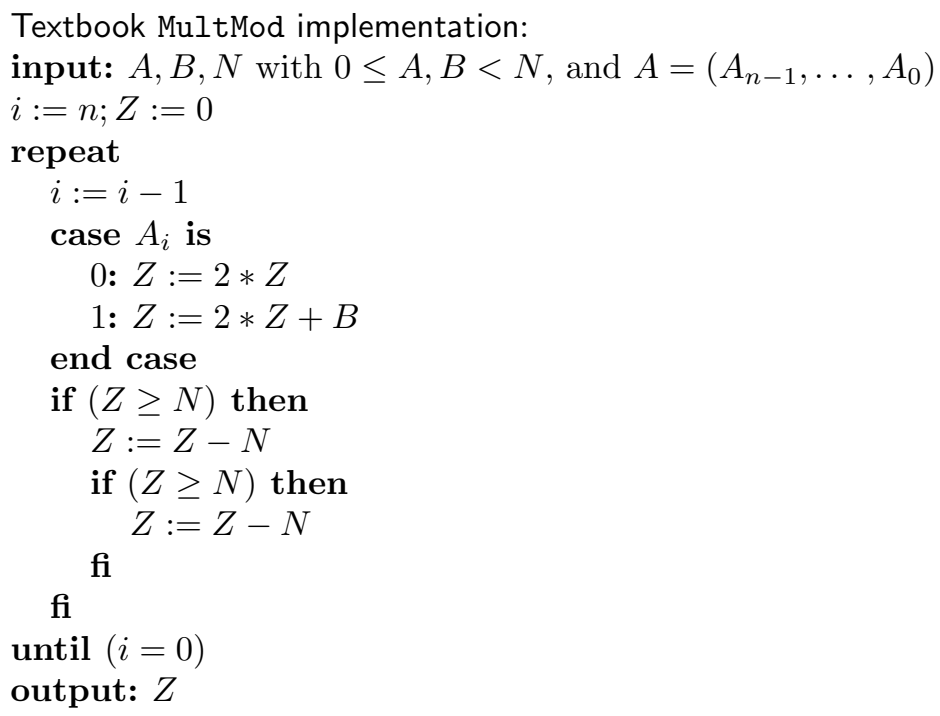

The extension is rather trivial. During the modular multiplication we simply have to "count" the number of subtracted $N$ 's. Observe that during a modular multiplication this implicit information is always known to the algorithm/hardware.

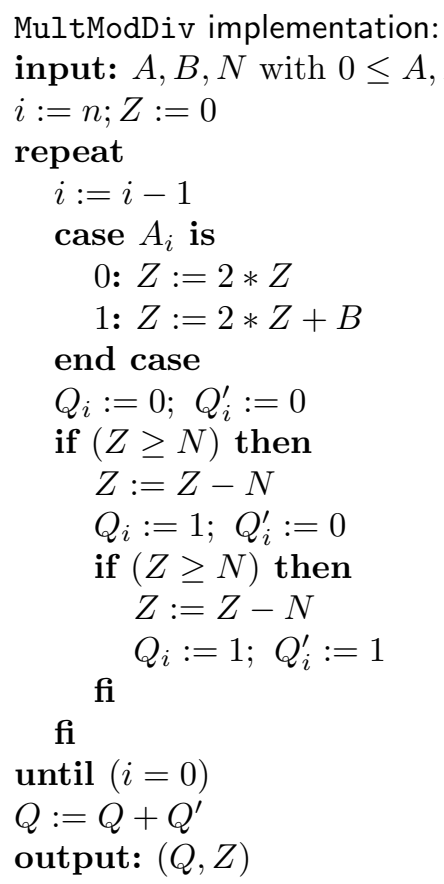


In the paper's full version we will actually show how the former algorithm can be simply integrated into the modular multiplication algorithm due to $\mathrm{H}$. Sedlak Sed.

The MultModDivInit ${ }_{n}$ and MultModInit ${ }_{n}$ are derived from the former ones essentially by exchanging the step $Z:=0$ with $Z:=C$.

\section{Conclusion}

In this paper we have introduced new efficient algorithms to compute $2 n$-bit modular multiplications using only $n$-bit modular multiplications. Using the

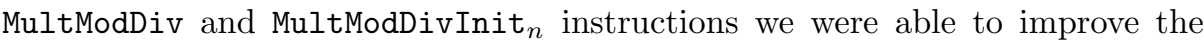
results presented by Pailler Pai]. The question of what is the minimal number of multiplications is still open, as we currently have no proof of the optimality of our algorithm.

Acknowledgments. We would like to thank Holger Sedlak for several valuable discussions on this topic.

\section{References}

[Ba] P. Barret, "Implementing the Rivest, Shamir and Adleman public-key encryption algorithm on a standard digital signal processor", Proc. of CRYPTO '86, Springer LNCS, vol. 263, pp. 311-323, 1987.

$\left[\mathrm{C}^{+}\right] \quad$ S. Cavallar et alii, "Factoring a 512 bit RSA modulus", Proc. of EUROCRYPT '00, Springer LNCS, vol. 1807, pp. 1-19, 2000.

[CQ] C. Couvreur, J.-J. Quisquater, "Fast decipherment algorithm for RSA public-key cryptosystem", Electronics Letters 18(21):905-907, 1982.

[DQ] J.-F. Dhem, J.-J. Quisquater, "Recent results on modular multiplication for smart cards", Proc. of CARDIS '98 Springer LNCS vol. 1820, pp. 336-352, 1998.

[HP1] H. Handschuh, P. Pailler, "Smart Card Crypto-Coprocessors for Public-Key Cryptography", CryptoBytes 4(1):6-11, 1998.

[HP2] H. Handschuh, P. Pailler, "Smart Card Crypto-Coprocessors for Public-Key Cryptography", Proc. of CARDIS '98 Springer LNCS vol. 1820, pp. 372379, 1998.

[Knu] D. E. Knuth, The Art of Computer Programming, Vol.2: Seminumerical Algorithms, 3rd ed., Addison-Wesley, Reading MA, 1999.

[MvOV] A. J. Menezes, P. van Oorschot, S. Vanstone, Handbook of Applied Cryptography, CRC Press, New York, 1997.

[Mo] P. L Montgomery, "Modular Multiplication without Trial Division", Math. of Computation, vol. 44, pp. 519-521, 1985.

[NMR] D. Naccache, D. M'Raihi, "Arithmetic co-processors for public-key cryptography: The state of the art", IEEE Micro, pp. 14-24, 1996.

[Om] J. Omura, "A public key cell design for smart card chips", Proc. of IT Workshop, pp. 27-30, 1990. 
[Pai] P. Pailler, "Low-cost double size modular exponentiation or how to stretch your cryptocoprocessor", Proc. of Public Key Cryptography '99, Springer LNCS, vol. 1560, pp. 223-234, 1999.

[Q] J.-J. Quisquater, "Encoding system according to the so-called RSA method, by means of a microcontroller and arrangement implementing this system", U.S. Patent \#5,166,979, Nov. 24, 1992.

[RSA] R. Rivest, A. Shamir, L. Adleman, "A method for obtaining digital signatures and public-key cryptosystems", Comm. of the ACM 21:120-126, 1978.

[Sed] H. Sedlak, "The RSA cryptographic Processor: The first High Speed OneChip Solution", Proc. of EUROCRYPT '87, Springer LNCS, vol. 293, pp. 95-105, 198.

[WQ] D. de Waleffe, J.-J. Quisquater, "CORSAIR, a smart card for public-key cryptosystems", Proc. of CRYPTO '90, Springer LNCS, vol. 537, pp. 503513, 1990.

[Wa] C. Walter, "Techniques for the Hardware Implementation of Modular Multiplication", Proc. of 2nd IMACS Internat. Conf. on Circuits, Systems and Computers, vol. 2, pp. 945-949, 1998. 